\title{
Experimental Equipment Research for Cryogenic Joule- Thompson Cryocoolers Comparison in IR Technology Sensors
}

\author{
Aleksandar Sicovic ${ }^{1, *}$ - Momčilo Milinovic ${ }^{2}$ - Olivera Jeremic ${ }^{2}$ \\ 1Le-tehnika, d.o.o., Slovenia \\ 2University of Belgrade, Faculty of Mechanical Engineering, Serbia
}

\begin{abstract}
This paper shows experimental setup for research of Joule-Thompson cryocoolers performances integrated in Dewar vessels with temperature detector simulator of IR sensor. Experimental solutions of transient temperature regimes of detector cooling on cryogenic cooldown temperature by using nitrogen as the coolant have been analyzed. In experiments of transient temperature influences, three principal methods of the fluid flow regulation, were compared. Results of experiments have shown important differences in quality and rate of cooling for IR detector integrated in cooling system with or without coolant flow regulation. The chosen thermodynamic regime parameters in experiments were suitable for the real necessities of detector cooling in IR sensor application. Scheme of the experimental equipment, its components, method of research and monitoring of desired transient function of temperature versus time in cooling (cooldown regime) of detector, and thermodynamic performances of the overall micro-cryogenic cooler are shown in this paper.
\end{abstract}

(C)2011 Journal of Mechanical Engineering. All rights reserved.

Keywords: cool-down regimes, Joule-Thompson micro-cryogenic coolers, sensitive element, cryogenic gasses, temperature measurements, experimentation of transient

\section{INTRODUCTION}

The basic purpose of cryogenic coolers is sustaining required temperatures of IR sensitive elements of different IR sensors like experimental sensitive camera, of the high resolution used in Infrared Thermographs method represented in paper [1], and other IR equipment, used in military and civil applications, [2] to [5]. Joule Thompson cryocooler is the representative type of this device and the most often used for these purposes. It is designed by dimensions of storage spaces which are usually, not more than several centimeters, and also to meet thermo-technical and general mechanical properties, necessary for rigid operational conditions. The device has to be operable inside the time interval, in different environmental conditions and under different external loads ranging between few Newton's up to several hundreds of Newton's. Furthermore, the Joule-Thompson cryocooler must provide the regulated temperature of cooling element inside the desired tolerance in the given time interval on the very low cryogenic temperature level, usually less than $100 \mathrm{~K}$, [6] to [11]. These requirements are further aggravated by the requirement of the desired temperature of IR sensors sensitive detectors achieved in a short time of less than a few seconds. The subject of this paper is a comparative analysis of tested transient temperature results, on the integrated laboratory assembly, presented in Fig. 1, the real designed Joule Thompson cryocooler, the real designed Dewar vessel, and an appropriate, experimental and designed simulation sample of temperaturesensitive element, as the model, of the real designed, expensive, mock-up detector.

The experimental set up of JouleThompson cryocooler assembly also includes the regulator and heat exchanger cooler packup, mounted in Dewar vessel, [12]. The image of the experimental, closed to real, design of the subassembly, used in this research is given in Fig. 1.

The regulator is a special subassembly that is separately integrated in the mockup Dewar vessel assembly with the heat exchanger module, [13], and joined with the detector simulator setup, which is all mounted in the internal space of cooler assembly. The aim of the test was to study the

*Corr. Author's Address: Le-tehnika, d.o.o., Šuceva 27, Kranj, Slovenia, aleksandarsicovic@yahoo.com 
optimum conditions of regulated or unregulated cooldown transient temperature regimes on the cooling detector sample as the model with properties that the real sensitive element requires in operation.

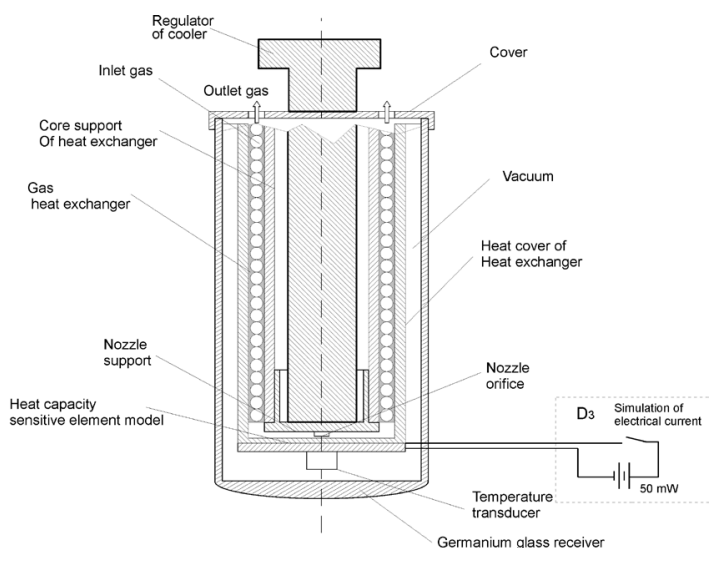

Fig. 1. Design of Dewar model vessel

The difference between the mock-up experiment assembly, Dewar vessels with coolers and the original assembly used on IR sensors is in the sample taken as the sensitive element instead of real sensors special elements determined by the same requirements of temperature conductivity. Emulation of detectors cooling rate on the mockup assembly is achieved by the specific heat capacity of sensitive element sample, rearranged by the calculation with the sample thickness. This corresponded to the effect of temperature conductivity rate, which was same as on the special element of real detector. Additional variations in experimental heat exchanges on the detector in the simulation process of Joule heat consumption rates, is also designed in experiments, (marked with D3 subassembly, Fig. 1). This was controlled by electric powered source, of $50 \mathrm{~mW}$ DC power supply, connected on the sample of sensitive element, made of prepared copper. Dewar vessel model, with JouleThompson cryocooler and nitrogen as coolant is universally used as an assembly for research of variable regulated or unregulated test conditions, required by sensor temperature sensitivity performances. The conditions are emulated by sensitive element through the loop D3. Experimental testing of regulated and unregulated coolers was implemented by modifying the subassembly of cooler regulator, in the same unique, and universally designed, Dewar vessel. Experimental research of the cooling performance test on the assembly using two representative regulator types inserted in the unregulated cooler assembly to compare the cooling and coolant efficiency designed in the same manner with modified regulation functions. The representative

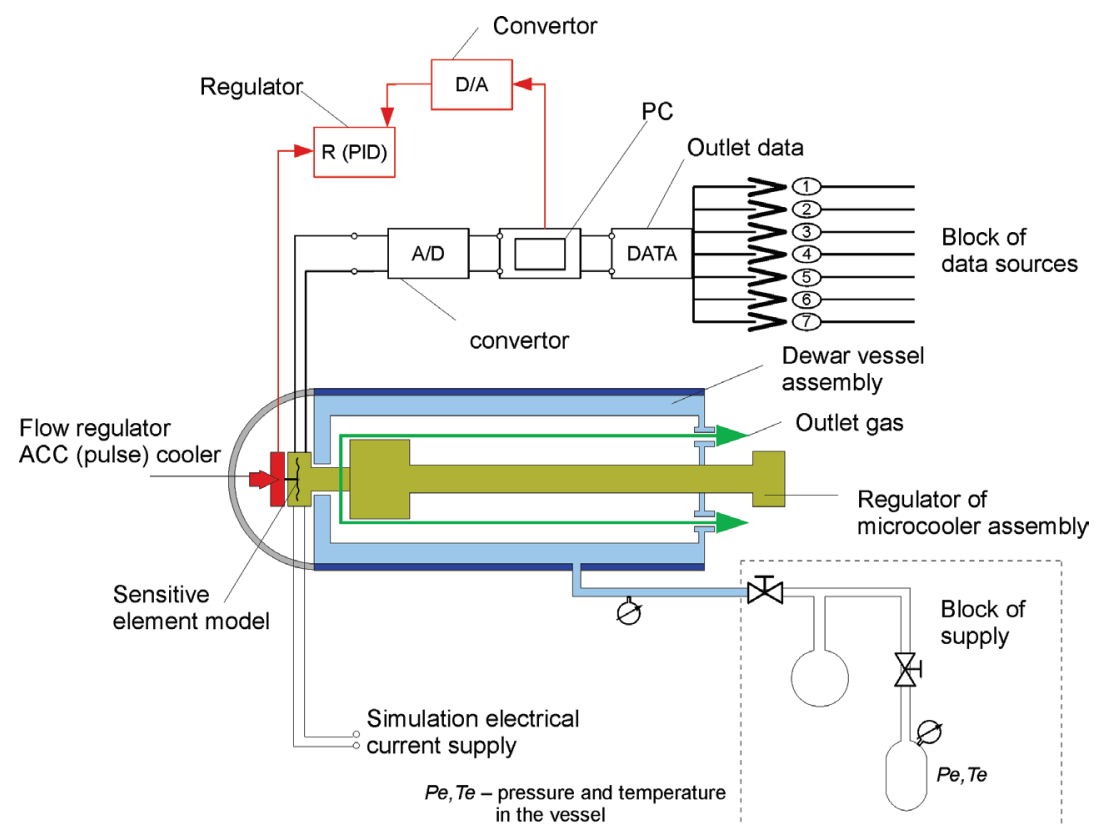

Fig. 2. Measurement flow chart 
regulator control subassemblies used here were the continuously designed self-regulation type, and pulsing coolant flow regulation type. All tested cooler types used nitrogen as the coolant.

\section{EXPERIMENTAL ASSEMBLY}

The setting method of experimental research of the universal Dewar vessel mock-up assembly, with cooler and emulation detector, in terms of different exchangeable coolant flow regulators, is given in Fig. 2.

A similar attempt has been done in the papers, [14] to [15], by Alexeev et al. and Luo at al., regarding mixed coolant and mixed JouleThomson cycle for different micro-coolers types.

The equipment consists of:

- Controlling block equipment and following data;

- Coolant supply control block equipment with capability to control;

- Temperature sensor for detector temperature measurements;

- Emulation detector with DC current supply;

- Dewar vessel assembly with Joule-Thompson cryocooler;

- Coolant regulator block inserted in a JouleThompson cryocooler (exchangeable regulator). Block data between positions 1 to 7, Fig. 2 represents controlled functions and measured data. The control functions are set by equipment and measured data, the desired environmental conditions and the desired controlled temperature closed behind the detector. The desired detector temperature and its time development as the transient cooldown performance was the focus of testing in these experiments. The evaluation of the quality of the experimental assembly with and without the use of regulators, was based on the criteria of the measured temperature and their time derivation. The controlling block can be used with different numbers of input functions versus on the type of exchangeable regulators requirements.

The controlling block can also be used for managing of power supplies when the model of the Dewar vessel with cooler is used without the regulator in an unregulated cooling manner. The coolant control and energy resources is achieved on the equipment with the cryogenic gas, which is delivered to the Dewar vessel assembly through the cooler to the detector over the nozzle with fixed or variable cross-section, controlled by continuous or pulsing regulator or uncontrolled (in unregulated regimes). All cases of control functions and measured data integrated on the cryogenic gas equipment and mock-up Dewar vessel assembly with or without regulated JouleThompson cryocooler, are shown in Fig. 2.

Three types of measurements were performed:

- without regulators,

- with continuous flow regulation of coolant, and

- with the pulsing flow regulated coolant.

The source of coolant in all three cases, were pressure vessels with nitrogen under pressure (360 bar), volume between 150 to 700 $\mathrm{cm}^{3}$, and the system of supply valves that provides pressure decreasing vs time [16] to [17]. An additional source of electric power $50 \mathrm{~mW}$ was turned on to simulate the constant Joule heat flux on the detector. In the case of unregulated cooler, feedback measurement branch through the A/D converter and the PC is used only for collecting data regarding the behavior of temperature detectors in real time. The same flow chart of data was used also with the continuous regulator. Controls of flow function were analogous, in this case, from the value of differential pressure to the direct motion of flow rate executive body in the regulator, without using the $\mathrm{PC}$ in the loop. The integrated control of temperature measurement and coolant flow was realized with additional PID loop in which an analog signal of the measured temperature is returned back to the controller $\mathrm{A} / \mathrm{D}$ converter over the $\mathrm{PC}$ and $\mathrm{D} / \mathrm{A}$ converter in to the operating body of pulse regulator. PID then implements pulse movement of the executive body, which controls the flow, thereby PC was controlling the current value of flow. The methodological flow chart settings of the management experiment shown in Fig. 3 is similar to the equipment in paper [18].

The central block C, consists of the control loops for measuring the pressure and the surrounding temperature around the sensor (the Dewar vessel assembly with a cooler) and the sensor detector temperature $T_{d}$. Other measuring 


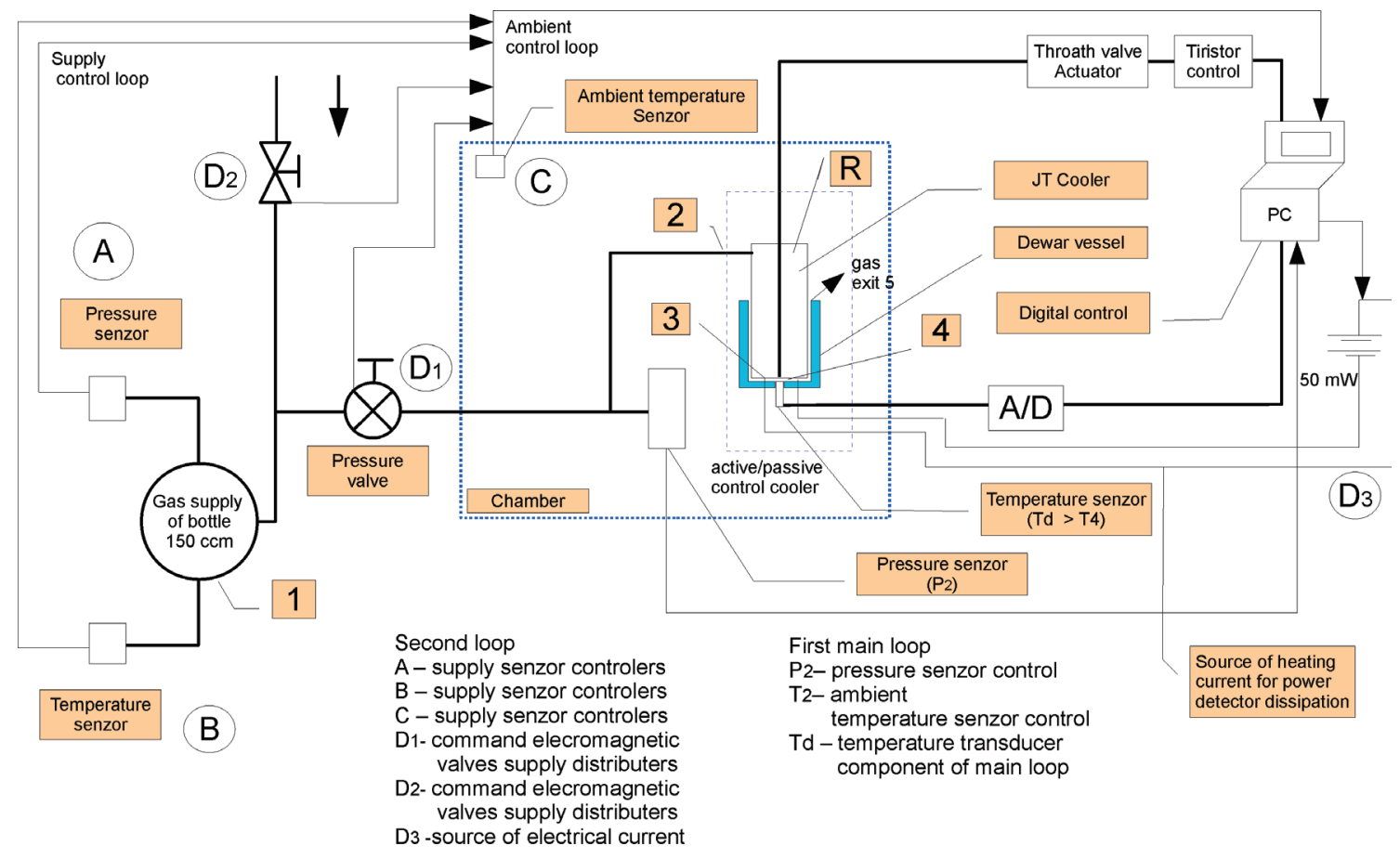

Fig. 3. Block diagram of instalation

and regulating loops, (secondary) are denoted as blocks A to D3. Their function is to control the coolant supply parameters. The integrated loops enabled the use of the module regulator $\mathrm{R}$ as a variable construction in the same experimental scheme. In this way it was able to examine the improvement of detector time and temperature characteristics, (sensitive elements), in a controlled or uncontrolled (unregulated) coolant supply. This experimental block diagram allows, for any modular, dimension, the adjusted design of Joule-Thompson cryocooler and the regulator $\mathrm{R}$ assembly to be used under the same conditions on the same equipment, with the same detector in the same Dewar vessel assembly.

It allows mutual comparison of the executive quality regulation of coolant flow. This equipment permits changing a JouleThompson cryocooler assembly that meets the required dimensional demands of Dewar vessel and particularly research of performances on the developing Joule-Thompson cryocooler using the same detector in the Dewar vessel. The regulation of power heat capacity of the emulated detector can also be achieved by changing the source of electric power in the electric circuit loop in certain limits.
By setting environmental conditions in Block C (appropriate exit temperature and pressure), it was possible to simulate the behavior of the detector in the required environment in which the real sensors could operate. The regulation of different coolant types is, also, possible on this equipment. The control of the operation mode in JouleThompson cryocooler and the sources of heat fluxes on real detector D3 can be regulated by a variation of pressure and flow of block $A, B$ by the commands D1 and D2. Hence, this equipment simulates all the real parameters of the IR sensor in the desired range of temperatures, either in the transient regime (cooldown), in the operating regime (run-time) or integrated in both modes. This universal equipment enables modular device exchange that emulates the specific conditions in which the Dewar vessel assembly, sensor and Joule-Thompson cryocooler operating as the key functional elements of cooled IR sensors.

\section{CONTROLS OF EXPERIMENTS AND THERMODYNAMIC FLOW CHART}

The thermodynamic cycle of the experiment consists of the process that changes 


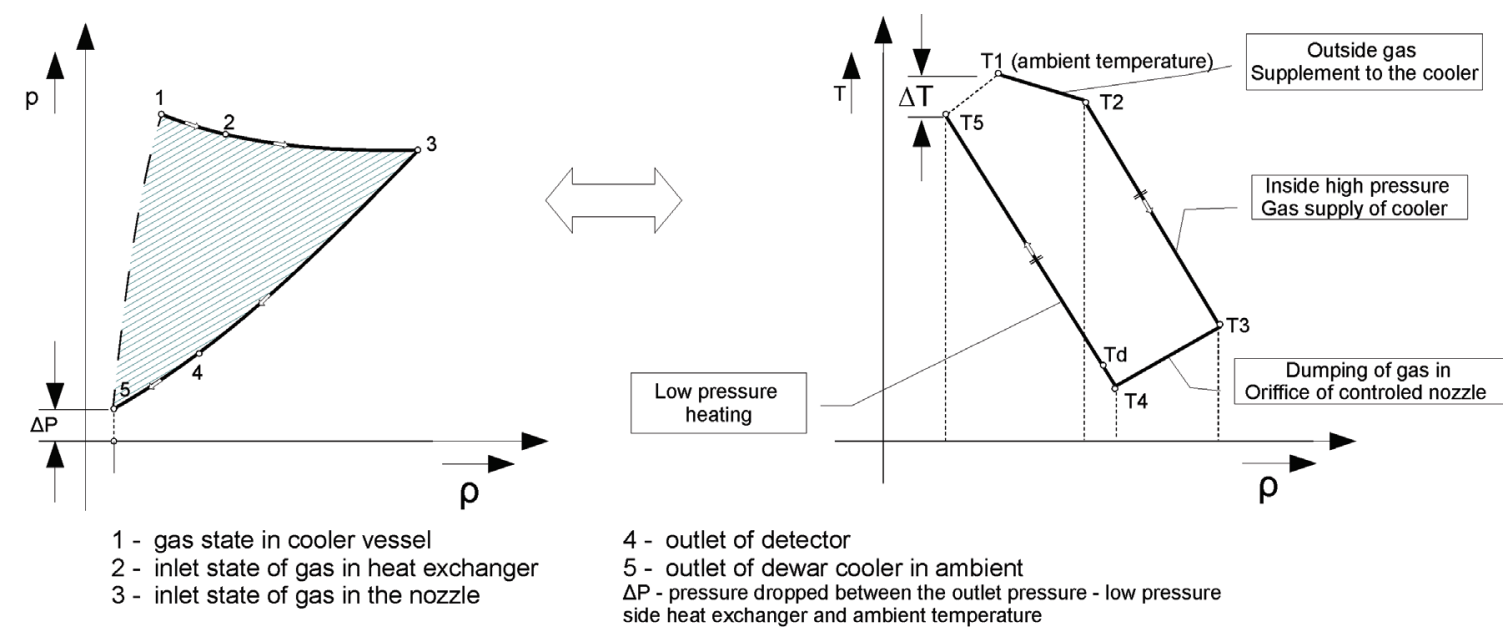

Fig. 4. Thermal cycle of cooled gas

the values of the coolant states. These values change from the input to the output values of the Dewar vessel assembly by the extreme gradients in overall thermodynamic state values (Fig. 2 and cycles in Fig. 4).

The most rapid change in the cooling cycle is the density of coolant, $\rho$, which extremely changes the gradient sign just in expected regulating point, T3 in Fig. 4, of Joule-Thompson cryocooler, making the system extremely sensitive by state values in this cycle point. The mass flow rate of coolant, as the regulation cooler performance, is mainly determined by density $\rho$; cycle overall, is control by pressure $p$, while, temperatures $T$, are only desired guided values of states in the cycle points. It was useful to present relationships between guidance and control major values of state $T$ and $p$, as the function of the main regulating thermodynamic value $\rho$, as graphical expressions of their thermodynamic changes in the cooling operations. It was also possible to make the process in the experimental testing set up visible by consequence on the mass flow disturbances intakes by the regulations over $\rho$, in the first proximity evaluations, in the rearranging guided and control $T$ and $p$ cycle values of state.

The quality of cooling and the procedure of thermodynamic control applied to the JouleThompson cryocooler in order to achieve the desired value of the cooling temperature on the detector, is shown in Fig. 4. The representation is based on the values of state equation for the cryogenic coolant taken from the input point of coolant state 2 to the output point coolant state 5 (Fig. 3), in the open coolant cycle. This means that the process of cryogenic coolant 1-2, corresponds to the state in the pressure vessel (state 1) and expansion up to the entrance of the Dewar vessel (state 2), and have not relevancing influences in cycle, if the supply pipeline is short. The next process 2-3, corresponds to the state of expansion of the cryogenic coolant in the cooler heat exchanger, while the expansion in the nozzle, which is regulated or unregulated, (fixed throat area), corresponds to the process $3-4$. State 4 is the input state of coolant in the free space of the detector, while the detector temperature $T_{d}$ is more or less equal to the temperature in state 4 . State 5 is the output state from the Dewar vessel to the environment (ambient conditions C, Figs. 3 and 4). Testing was realized for the three mentioned developing cases of integrated design sensor assemblies with or without the regulated coolant flow rate. An appropriately designed regulator was inserted in the same Dewar vessel and tested on the described universal equipment.

The experimental results of temperature decrease on the simulated detector $T_{d}$ (T4), are compared by rates in, the so-called, transient cooldown regime, as the main quality criteria for developing of sensor assembly. This criterion determines sensor readiness capabilities to start operating in the IR mode. In all the design cases mentioned above is the essential quality performance of the sensor as a feature for establishing rapid desired detector temperature 
of the sensor after the initiation of coolant flow. The rate of the detector temperature decrease, beyond process 3-4 in the thermodynamic cycle is presented in Fig. 4. The key parameter in this process is cryogenic coolant state magnitude, before and after this process. The requirements of each specified type of regulators is to provide the best behavior of process 3-4 in and after the transient temperature regime related to the requirement to achieve and to maintain the temperature point $\mathrm{T} 4$ in the operating regime. The regime of maintaining temperature is well known as the "run-time" [7], and depends on the above mentioned initial state of coolant magnitude. The Input flow in Joule Thompson cryocooler placed in Dewar vessel starts by the coolant state 2, Fig. 4 , and is finished by the output state 5 . Therefore, the flow which changes with the environment is directed by the conditions in the nozzle, process $3-4$, by the input and output state magnitudes of coolant. Changes of these magnitudes, which have different disturbances, in the case of regulated and unregulated coolant flow, controls quality of run time processes stabilization after cooldown transient regime also provide regulation possibilities to their diminishing, in run time temperature stabilization, after these unsteady state transients. This is essence item for any developing type subassembly, consists of Dewar vessel with Joule-Thompson cryocooler aimed for detector cooling purposes.

In the detector temperature transient cooldown decreasing phase, all the three developed assembly types studied in this research, had the same values of nozzle throat area and were only maximally opened in this regime. In all three cases of design subassemblies the coolant flow rate achieved maximum. Consequently, the coolant state magnitudes are also equal in all three cases, so the regulated and unregulated flow rate reached the critical regime in the nozzle throat. This means that the condition for the coolant is the same at any moment in time on the exit of the Joule-Thompson cryocooler and on the entrance to the detector during transient temperature decrease with a strong gradient. After reaching the desired temperature quickly, their further temperature regulation and stabilization, for the run time regime, expressed differences, depend of type of regulation, or consequences of unregulated mass flow processes. Achieving the critical regime in the nozzle, the mass flow becomes possible for regulation uses as in the critical flow with variable output magnitude states by variation nozzle throat cross section. However, the mass flow rate exchanges with the environment from detector volume in an aggravated form because of the mass accumulating in the free volume of the detector, and the mass flow regulation became sensible on the value of states in this free volume. For the optimization of the detector temperature rates of changes after the cooldown transient regime, and its temperature tolerance field necessary for running time process stabilization and control initiation, the coolant mass flow regulation is welcomed. The disturbances of temperature on the detector are aggravated by the fact that pressure and temperature in a detector free volume increases by the flow accumulation that causes weak detector cooling. The temperature difference of coolant and detector, therefore, decreases, and the heat flux is removed from a detector decreases.

\section{RESULTS}

The first impression is that there is an increase of the mass flow conveyed to the detector and that it can provide faster cooling and establishment of the desired temperature. The provided considerations joined with experimental experiences, have shown that the latter statement is only partially true, whereas the free volume of the cooler, where the detector and coolant are present, exchange the mass flow with the environment under thermodynamic leakage conditions. The conclusion is that any accumulation of the coolant mass, which is not regulated with the incoming flow, causes a temperature and pressure increase, in the part of volume space, where the detector is placed. Regardless of the increased mass in that space, temperature control by the heat exchange from the detector shows weaker performance. This was visible in case of the unregulated JouleThompson cryocooler, which required longer time for the stabilization of the desired temperature, and their further regulation for the run time regime (Fig. 5; curve 1).

Consequently, the exchange of heat with less consumption of coolant by flow rate, can achieved more rapid in run time temperature 
stabilization and their maintaining in the process. For the regulated coolers (curves 2 and 3) their time response sensitivities, controls accumulation of coolant in detector space, and by this feature, also control temperature differences between coolant and detector. The best control capabilities has pulsing regulator because of good short reaction time (curve 2, Fig. 5). Continuous regulated cooler have longer time response, because of analogous control loop. Consequently, its regulation capabilities allow gas accumulation in detector space, which makes transient detector temperature, toward run time stabilization regime, more unsteady (curve 3). All regulations are achieved by reducing the mass flow and avoiding the accumulation of gas in its free volume of the detector. Measurement error is considered summary for each unit assembly. Measurement results are presented with curves 1, 2 or 3, Fig. 5. The measurements were carried out under the same conditions in ten independent experiments. Diagrams of temperatures changes as a function of time and their represent mean values, of measured magnitudes, for one typical model sensor assembly, are given in Fig. 5. Using the same design cryocooler with mass flow regulator, (diagrams 2 and 3), the standard deviation of temperature measurements, in the cooldown mode, has not changed in relation to the diagram
1 , for unregulated cooler, and is approximately of $S T D E V=0.07$. It was determined by the end point of temperature, achieved in cooling versus time assumed as the end of cooldown mode taken as $T=100 \mathrm{~K}$, for all three cooler types. The relative error of a cooldown time, measured in relation to its average rate for all three types of model assemblies was less than $2.5 \%$. Values of error that appeared larger could be the result of structural dimensional tolerances of individual subassemblies which resulted in thermodynamic parameters error, and cannot be attributed to neither cumulative nor constructive errors. The represented cooldown regimes for advanced design solutions of cryocoolers often use two principals of coolant mass flow rate improvement to accelerate achievement of run time temperature in transient regime. This design solutions are known as double action flow [17] and demand flow, [17], [7]. The double action flow design type is not considered in this paper. The demand flow type corresponds to the designed case of regulated continuous type by bellows and is considered in this paper. The performances of all the mentioned design types depend on the coolant, on the initial run total pressure, on the designed nozzle, on the simulated heat silk on the detector, on the desired run time temperature and general overall thermal mass of the Dewar sensor vessel. These data

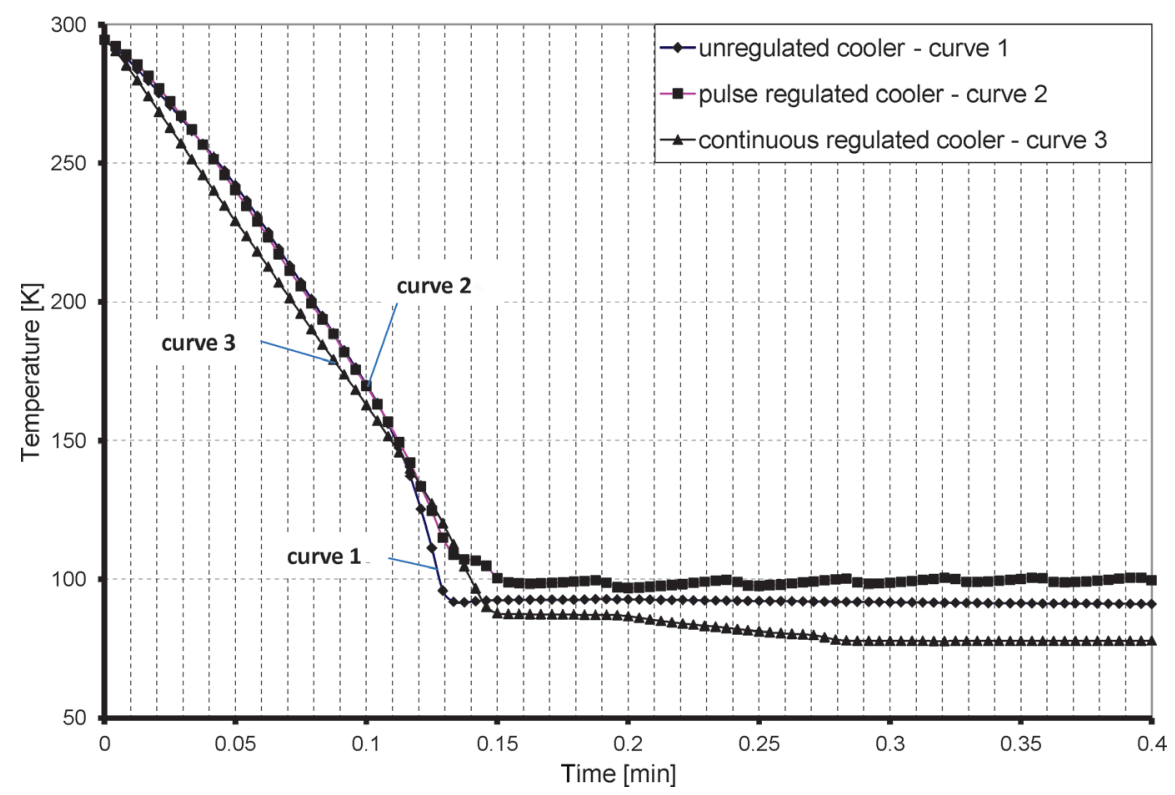

Fig. 5. Comparative measured temperature profiles versus time 
are not completely known in referenced papers. The nozzles with fixed, doubled and optimized demand flow, [17], Fig. 6, show more deviation of transient cooldown time caused by different mass flow rates operating in this regime.

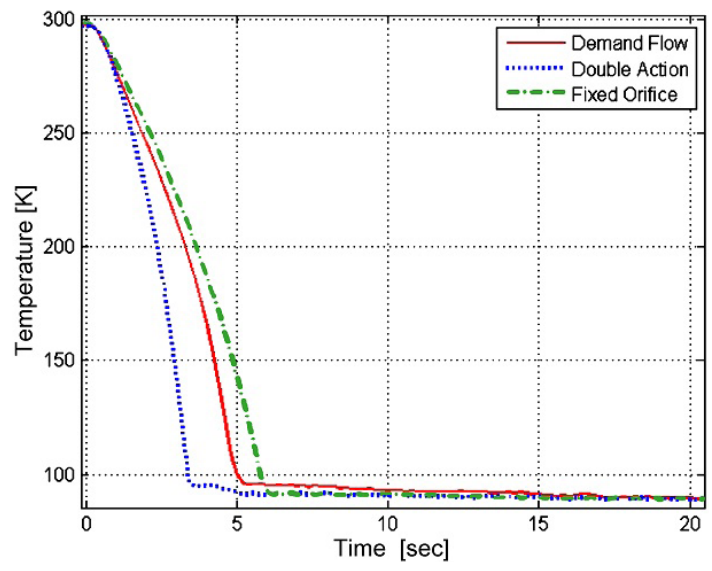

Fig. 6. Cool-down performance of a fixed-orifice cryocooler (dash dotted line), a demand-flow cryocooler (continuous line), and a double-action cryocooler (dotted line), [17]

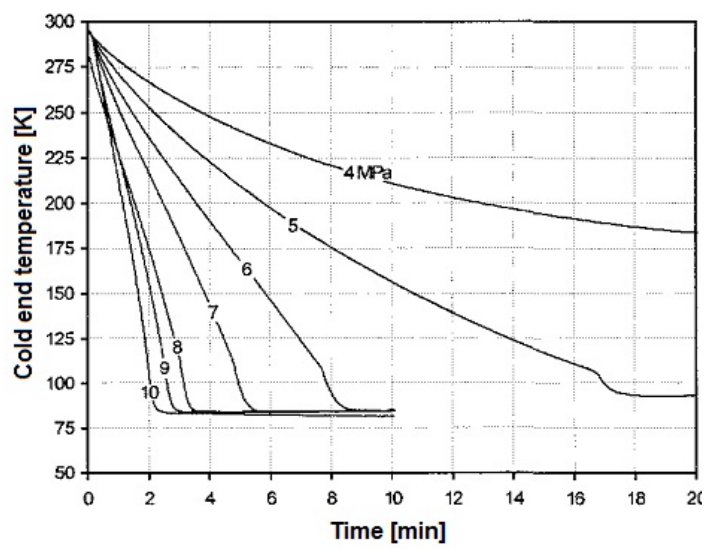

Fig. 7. Cooldown process of the Joule Thompson refrigerator with nitrogen at various pressures, [7]

The cooldown in Fig. 7 [7] shows variation dependant on pressure. These facts indicates more sensitivity of cooldown on the mass flow rate values. The cooldown temperature curves profiles in this paper, Fig. 5, are by form very similar to the experiments in papers [17], Fig. 6 and [7], Fig. 7, for both types of coolants, nitrogen and argon. Paper [7] uses nitrogen and also argon (not presented here) and [17] uses only argon as the coolant. As it is much cheaper in this paper, Fig. 5, only nitrogen coolant is used, for the achievement, of approximately the same run time temperature of about $100 \mathrm{~K}$. Cooldown pressures are much different in papers [7] and [17], and also vary from the cooldown pressure in this paper. The regulation of cooldown in the paper [7] is for the multipurpose cooldown initial starting of principally different sensor assemblies. The design presented in [17] is a single used cooldown system as in this paper. The above mentioned differences between this paper and [17] include novel designed pulsed regulation system of flow control. The heat sink is not comparable in these papers. This also points at differences in transient cooldown times for all design types, represented in Figs. 5 to 7. JouleThompson cryocooler systems, which choose cooldown regimes through advance designed nozzles, are able to control a steady state run time regime by a regulation system, independent of transient cooldown, for the required temperature, (Fig. 5). The relative measurement errors are not mentioned in the papers [7] and [17].

\section{DISCUSSION}

The measured performances of all the three Joule-Thompson cryocoolers design cases are the essential developing base for upgrading the unregulated sensors by regulated solutions of both regulation cases, continuous or pulsing types of regulators. Scientifically precise methodologies of transient performance simulation understand the comparison of transient regimes of the cooling to the same final temperature. The design of the regulator and Dewar vessel with a cooler assembly and an inserted detector simulator determines the equipment for the so-called cooldown regime testing, which is same but different in transient temperature stabilization for each of the cooler design variations. The gradient of the detector temperature on the decrease in the cooldown regime was the same in all tested cases, but cooldown time was different depending on the transient run time temperature stabilization.

This is different for all three types corresponding with the following explanations. The unregulated cooler continuously cools the 
detector in run - time (curve 1; Fig. 5) until the end of the full operation, and is not able to maintain a constant temperature. Tolerances of temperature changes in this transient run time temperature stabilization regime are acceptable for this type of sensor. The temperature instability in the run time regime was the consequence of the constant changes of exhaust conditions from the detector free volume during its cooling.

In the design case with continuous flow regulated cooler, transient regime is established on the desired temperature corresponding to the pressure difference between the environment and the free volume of the detector using an analog membrane pressure regulator. The operation in this regulator is based on the pressure differences between the environment condition and conditions in the free volume of the detector. Its reaction time is not rapid, and corresponds to the characteristic transient time (Fig. 5, curve 3).

Pulsing mode regulation is provided by using the direct detector temperature measurement loop. The loop is integrated over PC digital controller to change the amount of coolant flow through the nozzle and, control amount of the coolant mass in to the free volume space. This is achieved by using pulses of flow portions of the coolant, by opening and closing the throat nozzle in accordance with the continual leakage from the free volume in the environment, and to the cooling temperature $T_{d}$, measured on the detector. The method of achieving this temperature is accomplished by closed loop pulse flow regulation (Fig. 2, loop D/A-R) of PID controller type. Therefore, the transient regime measured to the same final temperature was not possible since the design of the process was conditioned by the assessment, considered temperature transient regime and their reaction time of detector. This is caused by detector free volume, and environmental relations, which were not independent of the regulation methodology.

Universal equipment provided the same sensor assembly with the Dewar vessel condition to be tested in terms of regulated or unregulated design solutions. In this sense, it was able to vary the thermodynamic cycle by changing the design and input or environmental parameters related to the cryocooler, or to the overall sensors. In further work on this equipment, experimental testing and theoretical simulations, the new features of coolants and new types of detectors for the extended requirements of modern sensors that operate in different environmental civil and military functional conditions would be expected.

\section{CONCLUSIONS}

This procedure provided unique experimental equipment and methods for measuring and checking the same functional parameters of the different model assemblies with the Dewar vessel joint with the Joule-Thompson cryocooler, regulator and IR detector. The testing equipment presented in this paper has the following appropriated unique performances:

1. Experimental integrated accessories are able to simulate environmental heat changes, reflected through the sensor focal plane array, as changeble heat sinks appeared in the real exploatation, Fig. 3, ambient control loop, and D3 loop. This capability is not expressed in equipment used [7] and [17].

2. Experimental integrated accessories, also have special properties to control mass flow rate of cryogenic coolant on the different type of the Joule-Thompson cryocoolers in the required critical flow point, Fig. 3, Tiristor PC control. This provides the possibility of testing for any of the designed cryocoolers type represented [7], [17] and [12]. The represented features provide for the testing of regulation effects for the mass flow rate control in the following: fixed orifices, demand flow types, double action types of cryocoolers, [17], [7], [12] but also provides the testing of special, regulated and digital controlled pulse types, novel designed and tested in this paper.

The method is applicable to both steady and unsteady transient temperature measurements of main subassembly functions for successful employment in the IR sensors.

The key question of the Joule-Thompson cryocooler efficiency is the heat transfer of two phase flow transformations of run time cooling and expansion through system inside of the Dewar vessel. Achieving temperature of detector, as the heat sink thermal mass through corresponding regimes, is a similar process to the two phase 
flow, the cooling method explained in [19]. The designed method is for the big PCM systems in environmental conditions. Considerations and model in paper [19], set up the question of how much intermediate obstacles influenced on the heat transfer efficiency, when the heat of phase transformations appears, and are they melting or does evaporation latent heat. For the cryocoolers, the latent heat is the main influenced performance linked with phase transformations of liquid to gas phase of coolant state. Heat transfer with appropriate obstacles of run two phase flows, rigidly influencing the phase changes exposing heat losses or heat sources depend on the coolant state. Paper [19] shows fin influences in the preserved flow system, on heat transfer cooling efficiency, in two phase flow condition. In the equipment presented in the cryocooler design, similar obstacles are avoided, to keep independant heat transfer in two phase flow processes, and to sustain unchanged state of liquid phase. The cooling duration of the emulated detector is the question of the fast two phase flow evaporation in the controlled gas environment as the heat transfer process from the coolant to the detector.

\section{ACKNOWLEDGEMENTS}

This paper is a part of research on the project Interdisciplinary Integral Research 47029 supported by the Ministry of Science and Technology of the Republic of Serbia in 2011.

\section{REFERENCES}

[1] Osterman, A., Dular, M., Hočevar, M., Širok, B. (2010). Infrared thermography of cavitations thermal effects in water. Strojniški vestnik - Journal of Mechanical Engineering, vol. 56, no. 9, p. 527-534.

[2] Semenov, V.I., Kuznecov, N.S., Bidenko, M.F., Lapunov, S.I., Komarov, N.B. (2004). Infrared radiation receiver. RF Patent, \#2 262776 C1.

[3] Knowles, P., Read. E. (2002). Infrared detector. UK Patent, \#2 368188 A.

[4] Hingst, U.G. (2006). Joule Thomson cooling apparatus comprising two counterflow heat exchanger. UK Patent, \#2 418479 A.
[5] Jim, K.J., Weber, D., Sagong, G. (2002). Two section gas purifier with Joule Thomson cooling device. US Patent, \#6 383259.

[6] Arkhipov, V.T., Borisenko, A.V., Getmane, V.F., Mikhalchenko, R.S., Povstiany, L.V. (1999). Long life Cryocooler for 84-90K. Cryocoolers, vol. 10, p. 467-473.

[7] Hong, Y.J., Park, S.J., Kim, H.B. (2004). The Performance of Joule Thomson Refrigerator. Cryocoolers, vol. 13, p. 497-502.

[8] Hong, Y.J., Park, S.J., Choi, Y.D. (2009). A Numerical Study of the Performance of a Heat Exchanger for a Miniature JouleThomson Refrigerator. Cryocoolers, vol. 15, p. 379-386.

[9] Chua, H.T., Wang, X., Teo, H.Y. (2006). A Numerical study of the Hampson-type miniature Joule-Thomson cryocooler. International Journal of Heat and Mass Transfer, vol. 49, p. 582-593, DOI:10.1016/j. ijheatmasstransfer.2005.08.024.

[10] Xue, H., Ng, K.C., Wang, J.B. (2001). Performance evaluation of the recuperative heat exchanger in a miniature JouleThomson cooler. Applied Thermal Engineering, vol. 21, p. 1829-1844, DOI:10.1016/S1359-4311(01)00050-3.

[11] Ng, K.C., Xue, H., Wang, J.B. (2002). Experimental and numerical study on a miniature Joule-Thomson cooler for steadystate characteristics. International Journal of Heat and Mass Transfer, vol. 45, p. 609-618, DOI:10.1016/S0017-9310(01)00165-X.

[12] Bradley, P.E., Radebaugh, R., Huber, M., Lin, M.H., Lee, Y.C. (2009). Development of a Mixed-Refrigerant Joule-Thomson Micro-cryocooler. Cryocoolers, vol. 15, p. 425-432.

[13] ter Brake, H.J.M., Lerou, P.P.P.M., Burger, J.F., Holland, H.J., Derking, J.H., Rogalla, H. (2008). Micromachined Joule-Thomson coolers for cooling low-temperature detectors and electronics. IEEE Sensors Conferences, p. 1352-1355.

[14] Alexeev, A., Haberstroh, Ch., Quack, H. (1999). Mixed gas J-T Cryocooler with Precooling stage. Cryocoolers, vol. 10, p. 475-479.

[15] Luo, E.C., Gong, M.Q., Zhou, Y., Liang, J.T. (1999). Experimental Comparison 
of Mixed-Refrigerant Joule- Thomson Cryocoolers with two types of counterflow Heat Exchanger. Cryocoolers, vol. 10, p. 481-486.

[16] Maytal, B.Z. (2000). Flow rate pressure dependence of a fixed orifice JouleThomson cryocooler. Advances in cryogenic engineering, vol. 45, no. I, p. 323-328.

[17] Tzabar, N., Lifshiz, I., Kaplansky, A. (2008). Fast cool-down J-T cryocooler to $88 \mathrm{~K}$.
Advances in cryogenic engineering, vol. 53B, p. 1025-1032.

[18] Arkhipov, V.T., Yakuba, V.V., Lobko, M.P., Yevdokimova, O.V. (1999). Multicomponent Gas Mixture for J-T Cryocooler. Cryocoolers, vol. 10, p. 487-495.

[19] Stritih, U., Butala, V. (2011). Energy savings in Building with a PCM Free cooling system. Strojniški vestnik - Journal of Mechanical Engineering, vol. 57, no. 2, p. 125-134, DOI:10.5545/sv-jme.2010.066. 